\title{
Preferable Wireless Sensor Networks Algorithm Based On the Maximum Weight Value of Time
}

\author{
Zhang Li \\ Software College \\ Shenyang Normol University \\ Shenyang, China \\ E-mail: 1098266067@qq.com \\ ZhaoYongyi \\ Software College \\ Shenyang Normol University \\ Shenyang, China \\ E-mail: 283631215@qq.com
}

\author{
Liu Yi \\ Software College \\ Shenyang Normol University \\ Shenyang, China \\ E-mail: 2843959961@qq.com
}

\begin{abstract}
The characteristic of Wireless Sensor Networks (WSN) is the one which supported by non-partition and noninfrastructure under large-scale self-organization and limited capacity. This kind of network system is selforganized by means of wireless communications between nodes. However, capacity within nodes is limited, thus we put forward a better method of cluster network on the basis of the maximum weight calculation. In order to make data transmission as effective as possible, we have adopted a method of broadcasting to classify node as inner-cluster node and cluster-head node, and found the longest path (also known as the critical path). Ultimately, network will find the cluster-head node of next layer quickly after the failure of one of the nodes under control, and it will make the valid node complete data transmission, achieve robustness of network, and maintain its life circle effectively.
\end{abstract}

Keywords--Wireless Sensor Network; Self-organization; Maximum Weight Value; the Longest Path;Robustness

\section{INTRODUCTION}

Wireless sensor network is made up of some minute nodes which were capable of computing and communicating in the monitoring area without security personnel. In light of the related environment, it can finish appointed tasks autonomously with "intellectual" capacity in monitoring network under self-control. It can be set up and released randomly, and the self-organizing network topology can be connected through wireless channel between nodes. It can be seen that there is strong cooperative capability among the nodes of sensor, which fulfilled tasks in overall situation through collecting data partially, pre-processing and data interaction between nodes. Usually, we use it in the way of multi-hop and peer to peer communications. Wireless sensor network can run independently, therefore it needs higher requirement on robustness of network topology. Since sensor node is capable of self-organizing as well as allocating and managing automatically, thus it can form a multi-hop wireless network system which transmits monitoring data automatically through topology control system and networking protocol. In the process of using wireless sensor network, some sensor nodes will lose their function due to energy depletion or other environmental factors, and some ones will be replenished into the network due to offset invalid nodes and incremental accuracy, thus the number of nodes hereof will increase or decrease dynamically thereby its topology will be changed accordingly ${ }^{[1-2]}$. As an important prerequisite, advanced network structure can make network enter an energyefficient working condition, and network topology is contingent on the networking routine which dispersed by the nodes which executive orders. When nodes lose their function partly in the process of energy depletion, physics damage and movement, topology control method can find it and activate corresponding countermeasure timely to ensure connectivity of the entire network. The merit of this method shall realize connectivity of network by node with the maximum weight value to offset the invalid node.

Some components, such as multi-leveled equations, graphics, and tables are not prescribed, although the various table text styles are provided. The formatter will need to create these components, incorporating the applicable criteria that follow ${ }^{[2]}$.

\section{BACKGROUND ANALYSIS}

Wireless sensor network is a kind of limited system of resource. It is extremely limited regard to battery capacity, processing capacity, and storage capacity and communication bandwidth. In the highly cover degree wireless sensor network, it is found that some overlaps exist in the area of perception close to nodes which make the information collected from adjacent node similar or form redundant data. If the base station received perception data dispatched from each node then it will lead much more energy consumed of nodes by wasting communication bandwidth, accordingly shorten network life circle. Furthermore, communication efficiency will be decreased by adding up the probability of data impact and data packet dropout as many nodes simultaneously transmit data which increase the dispatching difficulty of data link ${ }^{[5-6]}$. 
Due to the special nature of sensor network applications, the instability of wireless channel and the characteristics of energy constrained, the probability of damage of sensor network nodes is much greater than that of traditional network nodes, so the robustness of ad hoc networks is necessary to ensure that the loss of the sensor network will not affect the global task. Wireless Sensor Network is usually supplied by battery-Power and placed somewhere with no cadre, e.g. Plaees that People can not arrive at orareas that are dangerous. The ommunieation a bility of nodes is very limited. Therefore the sensor nodes must possess the self-organization ability to form amultihop wireless network for transmitting of monitoring data [7].

Wireless sensor network has a very broad application prospect. A traditional flooding algorithm is presented in the paper ${ }^{[4]}$. A node sends a message in the form of a broadcast. The node receives the message and then forwards it to all its neighbors. The process is repeated until the packet reaches its destination or reaches a maximum weight (i.e., the maximum time).For solving problems above mentioned, we will adopt WSN as an example to find out the most effective transmission path through the largest weight value, to fully explain there will be data redundancy of the adjacent sensor node from the same level. If one node have happened fails, and other nodes in the same level can give full play to their own role, we will use one to ensure the robustness of the network as the other one break down. This will make the transmission effectively.

\section{THE ALGORITHM BASED ON WEIGHTS}

Network with a simple activity represented AOE network of wireless sensor networks. AOE network is a weighted connected graph and the weight stands for duration. In order to achieve efficient transmission, it's necessary to find the longest path of WSN, that is the critical path, since there are multiple paths in data transmission in $\mathrm{AOE}^{[8]}$ network.

\section{A. Process of WSN Mante}

Analyzing flowchart of self-organizing sensor nodes according to the characteristics of it, shown as below:

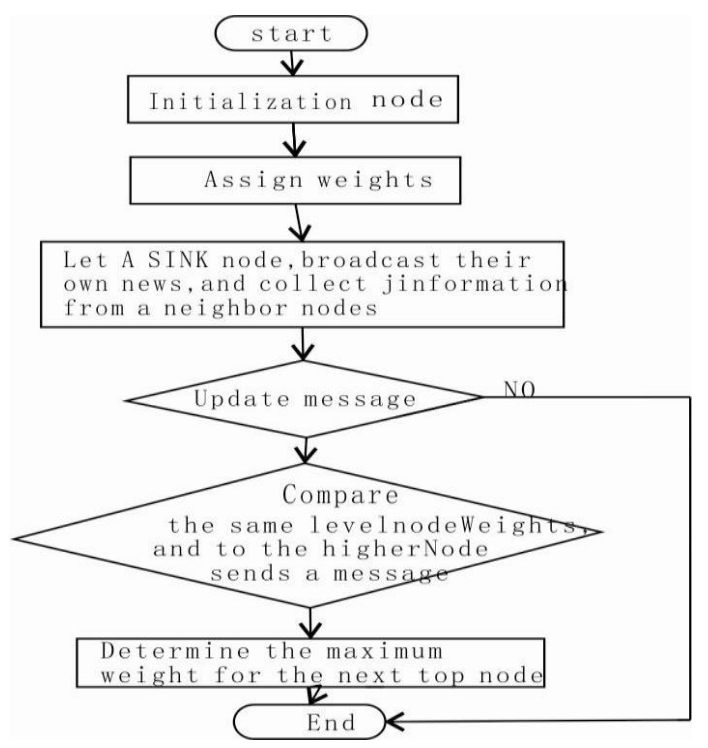

Figure 1. WSN Self-organizing Flow Chart

\section{B. Description of WDCA Algorithm}

By analyzing the process of WSN MANET, this section presents an algorithm based on WDCA ${ }^{[12]}$ weights. The algorithm considers the weights of the node and the effective time to complete the data transmission, data information of neighbor node in the same level, and can select the sensor note of the next-hop path based on the node data in the same level quickly.

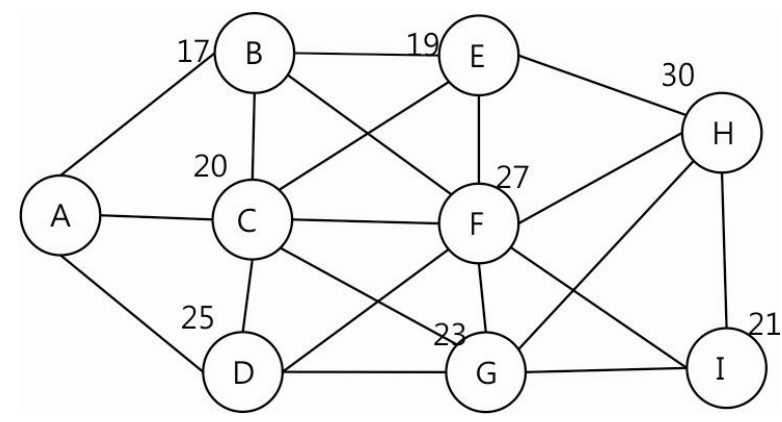

Figure 2. WSN network

\section{The algorithm Network robustness}

1. The length of the longest path starting from cluster head $\mathrm{A}$ to the destination node is the earliest time of occurrence. This time determines the overall $\mathrm{Ai}$, the earliest start time of transmission data, which starts from cluster head A. You can also define a latest start time as Li. Li-Ai means remaining time to complete network transmission. WSN topology in the transmission are all key transport route, therefore to finish key activities ahead cannot speed up data transmission ${ }^{[8-9]}$.

2. The length of the longest path starting from cluster head A to the destination node is the earliest time of occurrence. This time determines the overall $\mathrm{Ai}$, the earliest start time of transmission data, which starts from cluster head A. You can also define a latest start time as Li. Li-Ai means remaining time to complete network transmission. WSN topology in the transmission are all key transport route, therefore to finish key activities ahead cannot speed up data transmission.

For WSN path of effective transmission, it is critical path in AOE network as an example for following analysis:

\section{1) Critical path analysis}

In the AOE network to identify the critical path to find the $\mathrm{Ai}=\mathrm{Li}$, in order to get the $\mathrm{AOE}$ network in the activities of $\mathrm{Ai}$ and $\mathrm{Li}$, at first we should get the earliest time VA (j) and the latest time VL (j).We put each link of transmission by the $\operatorname{arc}<\mathrm{j}, \mathrm{k}\rangle$, its duration is dut $(\langle\mathrm{j}, \mathrm{k}\rangle)$ recorded as follows:

$$
\begin{aligned}
A \mathrm{i} & =V A \mathrm{j} \\
L \mathrm{i} & =V L k-\operatorname{dut}(<j, k>)
\end{aligned}
$$

\section{a) Solving VAj and VLj need to be divided into two} steps

From the VA $(0)=0$ start to push forward 
$V A(j)=\operatorname{Max}\{\operatorname{VA}(j)+\operatorname{dut}(<i, j>)\},<i, j>\in T, \mathrm{j}=1,2, \cdots n-1$

Among them, $\mathrm{T}$ is the collection of all the arcs with the $\mathrm{j}$ node as the head. back

b) From the VL (n-1) $=V A(n-1)$ start to push the

$V L(i)=\operatorname{Min}\{V L(j)-\operatorname{dut}(<i, j>)\},<i, j>\in s, i=n-2, \cdots 0$

And $\mathrm{S}$ is a collection of all the arcs the tail of i node.

The calculation of these two recursion formulas must be carried out in the premise of the topological order and the inverse topological order. The VA $(\mathrm{j}-1)$ necessary to $\mathrm{Vj}$. Determine all the earliest time occurrence of all the precursors, and $\operatorname{VL}(\mathrm{j}-1)$ then it is necessary to determine $\mathrm{Vj}$ the most of the later time of the subsequent occurrence.Thus VA(j-1) and $\operatorname{VL}(\mathrm{j}-1)$, it can be calculated on the basis of the topological sort.

2) The algorithm design

Input $\mathrm{n}$ arc $(\mathrm{j}, \mathrm{k})$, The storage structure of $\mathrm{AOE}$ net is established.

From the SINK node start,in order to $\mathrm{VA}[0]=0$, according to the topological order of the other nodes, the earliest time of occurrence $\mathrm{VA}[\mathrm{i}](1 \leq i \leq n-1)$. If the number of vertices in the ordered sequence is less than the number of vertices $n$ in the network, the existence of the network cannot find the critical path, the algorithm terminates;

Starting from the sink point Vn,order to VL[n$1=\mathrm{VA}[\mathrm{n}-1]$,According to the inverse topological order, find out the vertices latest time $\operatorname{VL}[i](n-2 \geq i \geq 2)$;

According to each vertex $\mathrm{VA}$ and $\mathrm{VL}$ of the value,Find the earliest start time $\mathrm{A}(\mathrm{s})$ and the latest start time L(s) of each arc S. If an arc satisfies the condition $\mathrm{A}(\mathrm{s})=\mathrm{L}(\mathrm{s})$, it is the key activity, as mentioned above,calculate the value of each vertex is in the process of topological sort, the need for topological sort algorithm for the following changes:(1)An initial value which is set before the topological sort, $\mathrm{VA}[\mathrm{i}]=0(0 \leq \mathrm{i} \leq \mathrm{n}-1) ;(2)$ In the algorithm, an algorithm is added to calculate the direct successor of the earliest occurrence time of operation:if $\mathrm{VA}[\mathrm{j}]+\operatorname{dut}(\langle\mathrm{j}, \mathrm{k}\rangle)>\mathrm{VA}[\mathrm{k}]$, thenVA[k]=VA[j]+dut $(\langle\mathrm{j}, \mathrm{k}\rangle))$ (3)In order to in reverse topological order sequence order calculation of each vertex of the VL value, down in the topological sort of the process obtained the topological order sequence.

3. A node is treated as SINK node, other nodes are in undetermined condition except the initial SINK. Suppose SINK node cluster network launched the first round of the message, $\mathrm{B}, \mathrm{C}, \mathrm{D}$ node receiving the message, the $\mathrm{B}, \mathrm{C}, \mathrm{D}$ node According to the information received, update their data tables to change their status to cluster member status, and broadcast their own news cluster members, so the A, E, F, G will have been received by the node B, C, D three Broadcast messages nodes adjacent nodes, A node SINK node, it does not process the message, and E, F, G node according to news collected from a neighbor node, compute their weights, weights and compares the same level and always save energy value larger node node for the next hop path. Since the energy F node maximum effective longest, and $\mathrm{D}$ adjacent nodes than the $\mathrm{B}, \mathrm{C}$ right big value, then finalize the $\mathrm{D}$ node to the next hop path node, then broadcasts its own cluster head message. Thus $\mathrm{B}, \mathrm{C}, \mathrm{E}, \mathrm{F}, \mathrm{G}$ can receive the broadcast message, since the B, C, D cluster member status has been determined, it does not process the message, and E, G collected in accordance with the calculated value of the message and the right to change their status as a member state of the cluster, and the cluster members broadcast their message. In this case H, I node at the same level, the same as management station (final receiving node). $\mathrm{H}$, I node status is determined in accordance with the rules to change their status as a member state of the cluster, and the cluster members broadcast their message, it will receive $E, F, G$ node broadcasts the message, then $\mathrm{H}$, I node in accordance with the income the information, compare their weights, then finally selected as the next hop node $\mathrm{H}$ transmission node, thus generating a network clustering results as shown below.

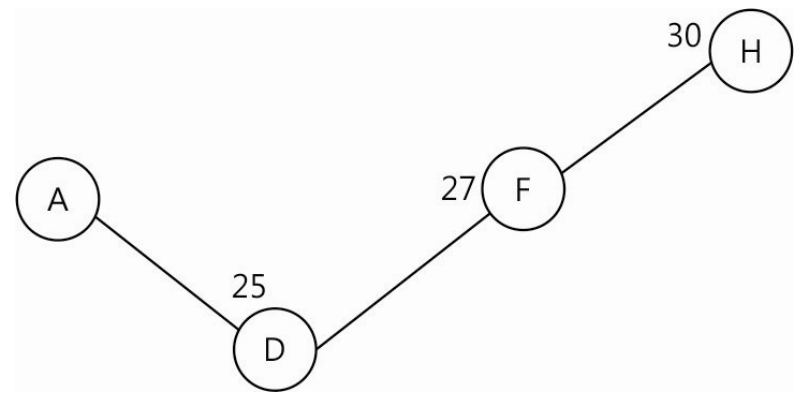

Networking Results

\begin{tabular}{|l|c|c|c|c|}
\hline NODE & A & D & F & H \\
\hline VALUE & SINK & 25 & 27 & 30 \\
\hline
\end{tabular}

\section{Algorithm when a Node Loses Effectiveness}

As shown in Fig 1.2, if node D in the WSN fails, all links and nodes connected to the D (dotted line) are expired, then, as shown in Figure 1.3, as neighbor notes that are in the same level as D, note B and C has and the same information as note $\mathrm{D}$. Since node $\mathrm{C}$ has a greater weight than the node $\mathrm{B}$, node $\mathrm{C}$ as the next hop transmission node is added to the critical path instead of $D$ [9-10]. Through carrying out the transfer procedure to get the the critical transmission path as shown in Figure 3.

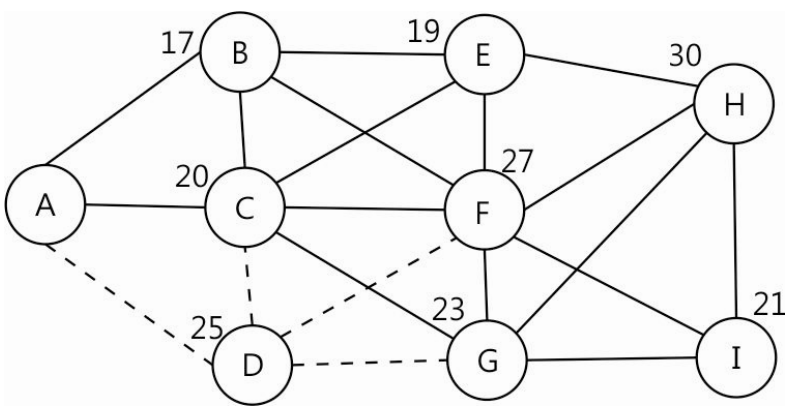

Figure 3. D node failure 


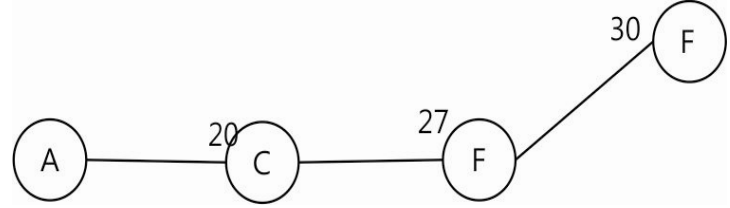

Figure 4. C node joins the critical path

\section{CONCLUSION}

This paper proposed the time needed to complete the whole data transmission through the maximum weight according to self-organizing capability for wireless sensor networks. Furthermore, it showed that shorten the time couldn't complete the task ahead or find the longest path (that is, critical path) to extend effective life cycle of the network. Otherwise, the maximum weights can find the next hop transmission node quickly for invalid node and after a node fails, the other can be filled soon to ensure network connectivity.

In order to effectively solve the wireless sensor network maintenance difficult problem, networking algorithm with better performance is an effective method to solve the problem. The paper presents the architecture of wireless sensor network model and its connectivity is defined, then the paper presents a networking algorithm for wireless sensor networks with good connectivity.

\section{REFERENCES}

[1] Bao L,Garcia-Luna-Aceves J J. Topology Managerment in ad hoc Networks[C]//Proc 4th ACM Int'l Symp on Mobile Ad Hoc Networking\&Computering(MobiHoc2003),Annapolis,Maryland.20 03.129-140.
[2] C.Intanagonwiwat,R.Govindan,D.Estrin.Power Aware Routing in Mobile Ad Hoc Networks. IEEE/ACM MobiCom, 2002, 622-628

[3] AKYILDIZ I F, SU W, SANKARASUBRAMANIAM Y,etal.Wireless sensor networks:a survey[J].Computer Networks, 2002,38:393-422.

[4] Kulik J, Heinzelman W,Balakrishnan H. Negotiation based Protocols for Disseminationg Information in Wireless Sensor Networks[J]. Wireless Networks, 2002, 8(2-3):169-185.

[5] G.J. Pottie and W.J.Kaiser, wireless Integerated network sensors, comm.ACM, 2000,43(5): 51-58

[6] Akyildiz I F,Su W,Sankarasubramaniam Y,et al. Wireless sensor networks:asurvy. Computer Networks, 2002,38(4):393-422

[7] H LUO, FAN YE, J CHENG, S LU, L ZHANG.TTDD: A Twotier Data Dissemination Model for Largescale Wireless Sensor Networks. Wireless Networks, 2005, 11(2):161-175.

[8] Yan Yumin, Wu Weiming. Data structure (C language structure) Tsinghua University press 2007

[9] Yao K. Sensor networking: Concepts, applications, and challenges [J]. Acta Automatica Sinica, 2006, 32(6): 839-845.

[10] S. Lin, J. Zhang, G. Zhou, L. Gu, J. A. Stankovic, and T. He. Atpc: adaptive transmission power control for wireless sensor networks. ACM Sensys, 2006.

[11] Taherkordi A.,Taleghan M.A., Sharifi, M.Achieving availability and reliability in wireless sensor networks applications Availability, Reliability and ecurity,2006.ARES 2006.In: The First International Conference on 20-22 April 2006 57 62.

[12] . Shikai Wang,Bo Yin, Yanping Cong. Performance Evaluation of SCTP Multipath Parallel Transmission-Based on WCDMA Channel and WLAN Channel[C]. The 15thIEEE International Conference on Communication Technology(ICCT 2013), Guilin, China, 2013:757-764

[13] Craig Ulmer. Wireless Sensor Networks[EB/OL].users.ece.gatech.edu/ grimace/research/sensorsi mii/. 